\title{
Efeitos do posicionamento e quantidade de prática mental na aprendizagem do arremesso do dardo de salão
}

\author{
Thábata Viviane Brandão Gomes \\ Herbert Ugrinowitsch \\ Robledo Rodrigues Coelho \\ Nádia Fernanda Schmitt Marinho \\ Fabiano de Souza Fonseca \\ Rodolfo Novellino Benda
}

Escola de Educação Física, Fisioterapia e Terapia Ocupacional, Universidade Federal de Minas Gerais, Belo Horizonte, MG, Brasil

\begin{abstract}
Resumo: A prática mental parece contribuir no processo de aprendizagem motora, mas ainda não estão claros os efeitos do posicionamento e da quantidade de prática mental em relação à prática física, sendo este o objetivo do presente estudo. Setenta e dois universitários de ambos os sexos e novatos na tarefa foram distribuídos em seis grupos de prática combinada (física e mental) para a aprendizagem do arremesso do dardo de salão. Na fase de aquisição, os sujeitos realizaram 50 tentativas de prática física e 18, 90 ou 180 tentativas de prática mental (conforme cada grupo) antes ou após a prática física. Dez minutos após a fase de aquisição foram realizados os testes de retenção e transferência. Não foi observada diferença significante entre os grupos nos testes. Os resultados indicaram que a quantidade e 0 posicionamento da prática mental não influenciaram a aprendizagem do arremesso do dardo de salão.
\end{abstract}

Palavras-chave: prática mental; prática física; quantidade de prática mental; posicionamento da prática mental.

\section{Effects of position and amount of mental practice in dart saloon throwing learning}

Abstract: Mental practice seems to contribute to the motor learning process but it is still not clear the effects of position and amount of mental practice in relation to physical practice, which was the aim of this study. Seventy two undergraduate students of both sexes and novice in the task were assigned in six groups of combined practice (physical and mental) for learning the dart saloon throwing. In acquisition phase, subjects performed 50 trials of physical practice and 18,90 or 180 trials of mental practice (according to each experimental group) before or after physical practice. Ten minutes after acquisition phase it was performed retention and transfer tests. The results showed that amount and the position of mental practice did not influence dart saloon throwing learning.

Keywords: mental practice; physical practice; amount of mental practice; temporal location of mental practice.

\section{Introdução}

A prática mental tem sido tradicionalmente testada quando comparada com outros tipos de prática. Além dessa questão, ela tem também sido estudada a partir da investigação de variáveis como duração das sessões, quantidade de prática, nível de habilidade dos sujeitos, diferenças individuais, tipo de tarefa, combinação de prática (física e mental) (ETNIER; LANDERS, 1996; RICHARDSON, 1967b; STEBBINS, 1968; WEINBERG, 1982; WEINBERG et al., 1991).

Ao analisar o tempo de duração de prática mental, Richardson (1967a) observou, em revisão de estudos desenvolvidos entre 1936 e 1963, que um tempo prolongado foi prejudicial ao desempenho do esportista. Foi sugerido então um tempo máximo de cinco minutos sem pausa para descanso. Mackay (1981), em estudo posterior, chegou aos mesmos resultados encontrados por Richardson (1967a). Entretanto, Weinberg (1982) revisou estudos em um período maior (entre 1916 e 1981) e verificou que o tempo de duração deve depender tanto da natureza da tarefa (habilidades simples ou complexas; habilidades simbólicas ou motoras) quanto das características do indivíduo (nível de habilidade na tarefa, capacidade de imaginação e experiência prévia). Em uma revisão dos trabalhos realizados entre 1934 a 1981, Feltz e Landers (1983) observaram melhoria no desempenho de tarefas cognitivas após um período de curta duração. Por outro 
lado, em tarefas motoras foi identificada melhora apenas após longo período de duração de prática mental. Apesar de vários estudos terem investigado o tempo de duração, Etnier e Landers (1996) sugerem que quantidade de prática pode ser uma variável mais importante que tempo de duração.

O posicionamento da prática mental em relação à prática física é outra variável estudada e também tem sido tradicionalmente testada com prática física e combinação de prática ( $\underline{\mathrm{CAHN}}$, 2008; $\underline{\text { HIRD }}$ et al., 1991; MACKAY, 1981; MILLARD et al., 2001; RICHARDSON, 1967a; STEBBINS, 1968; WULF et al., 1995). Este tema de investigação está vinculado aos resultados de pesquisa que investigaram 0 próprio efeito da prática mental e combinação de prática.

Os resultados encontrados em revisões de estudos e pesquisas de meta-análise têm concluído que a prática combinada e a prática física são mais efetivas que a prática mental, a qual, por sua vez, é superior à ausência de prática (DENIS, 1985; DRISKELL et al., 1994; FELTZ; LANDERS, 1983; LANDERS; LANDERS, 1973; RICHARDSON, 1967a, 1967b).

Em alguns estudos a prática combinada foi estruturada alternando as sessões de tentativas de prática física e mental (MACKAY, 1981; MARING, 1990; WULF et al., 1995). Os resultados mostraram em todos os estudos que tanto a prática física quanto a prática combinada levaram à aprendizagem da habilidade praticada. Porém, enquanto Mackay (1981) concluiu que os grupos de prática física e prática combinada foram similares, Maring (1990) e Wulf et al. (1995) verificaram que a prática física foi superior à prática combinada.

De outro modo, Stebbins (1968) investigou a combinação de prática utilizando dois grupos: GPMF (prática mental antecedeu à prática física) e GPFM (prática física foi realizada inicialmente). Ambos os grupos de prática combinada apresentaram desempenhos semelhantes e superiores aos grupos de prática mental e controle. Os resultados indicaram que 0 posicionamento das práticas não interferiu, visto que os dois grupos de prática combinada tiveram resultados similares.

Weinberg et al. (1991) e Etnier e Landers (1996) investigaram simultaneamente a duração da prática mental e seu posicionamento em relação à prática física. Eles utilizaram a mesma tarefa (arremesso do lance livre no basquetebol), população (sujeitos experientes), delineamento similar e encontraram resultados distintos. Em Weinberg et al. (1991) não foi encontrado efeito significante quando os sujeitos praticaram mentalmente a tarefa por um, cinco ou dez minutos, antes ou após a realização da prática física que durou três minutos.

Por outro lado, os resultados encontrados por Etnier e Landers (1996) indicaram superioridade dos grupos que primeiro realizaram a prática mental comparados aos grupos que primeiro realizaram a prática física. Segundo os autores, o posicionamento anterior da prática física pode ter facilitado o desempenho e com isso requerido menos esforço na sequência. Esta conclusão sugere uma superioridade da prática física em relação à prática mental. Sobre a duração, quatro diferentes tempos de prática mental foram investigados (um, três, cinco e sete minutos). Os grupos que praticaram por um e três minutos, independente de seu posicionamento, foram mais efetivos que os demais grupos. Tal resultado sugere que quanto maior a duração de prática mental, mais os sujeitos ficam entediados e se distraem. Em conjunto, os achados desses estudos mostram resultados distintos quanto ao posicionamento e quantidade de prática mental em relação à prática física, mesmo utilizando tarefa e delineamento semelhantes. Esta divergência evidencia a necessidade de novos estudos sobre a temática.

Mais recentemente, Cahn (2008) observou a combinação de prática avaliando os efeitos de distribuições variadas no desempenho de uma partitura musical. Os participantes eram músicos experientes e foram aleatoriamente distribuídos em grupos de prática física (PF), prática mental (PM), prática combinada 1 (66\%PF:33\%PM) e prática combinada 2 (33\%PF:66\%PM). Os resultados não apresentaram diferença significante entre os grupos de prática. Além disso, distribuições variadas de tempo de prática utilizadas nos grupos de prática combinada não foram relevantes para a aprendizagem motora. Este resultado não corrobora os achados que apontaram superioridade da prática combinada e prática física sobre a prática mental (MACKAY, 1981; MARING, 1990; STEBBINS, 1968; WULF et al., 1995). 
Considerando as contradições no resultados dos estudos sobre quantidade de prática mental e seu posicionamento na prática combinada, 0 presente estudo investigou os efeitos do posicionamento e quantidade de prática mental na aprendizagem do arremesso do dardo de salão.

\section{Método}

\section{Participantes}

Participaram do estudo 72 universitários voluntários, de ambos os sexos, com idade média de $24,2 \pm 4,2$ anos, com consentimento livre e esclarecido e sem experiência prévia com a tarefa em questão. Os procedimentos utilizados neste estudo foram aprovados pelo Comitê de Ética em Pesquisa da Universidade (298/05).

\section{Instrumento}

Foram utilizados seis dardos de salão com ponta metálica e um alvo circular com círculos circunscritos nas cores vermelha (centro), preta e branca, com $1 \mathrm{~cm}$ de raio, suspenso na parede com seu centro posicionado a $1,73 \mathrm{~m}$ de altura. A pontuação teve uma variação de 12 pontos (quando acertava o centro do alvo) diminuindo a cada faixa até 1 ponto no círculo mais externo. As execuções em que 0 dardo não atingia 0 alvo receberam pontuação zero.

\section{Tarefa e delineamento experimental}

A tarefa proposta foi arremessar dardos de salão com o objetivo de acertar o centro do alvo. Setenta e dois participantes foram aleatoriamente divididos em seis grupos experimentais em que se manipulou quantidade e posicionamento da prática mental ( $n=12)$ : 1) 18MF (18 tentativas antes da prática física); 2) 18FM (prática física seguida por 18 tentativas); 3) 90MF (90 tentativas antes da prática física); 4) 90FM (prática física seguida por 90 tentativas); 5) 180MF (180 tentativas antes da prática física); 6) 180FM (prática física seguida por 180 tentativas). Todos os grupos realizaram 50 arremessos (prática física) a uma distância de 2,37 $\mathrm{m}$ do alvo na fase de aquisição com a pegada preensão profissional, em que o dardo era apreendido entre os dedos com os dedos mínimo, médio e indicador de um lado e polegar e anular do outro lado. Dez minutos após a fase de aquisição, foi aplicado o teste de retenção seguido do teste de transferência. Para o teste de transferência, foram modificadas a distância $(3 \mathrm{~m})$ e a forma de preensão (pegada em " $\mathrm{V}$ ", em que o dardo se posicionou entre os dedos indicador e médio flexionados).

As diferentes quantidades de prática mental foram estabelecidas considerando o tempo efetivo de prática mental utilizado em outros estudos (ETNIER; LANDERS, 1996; WEINBERG, 1982; WEINBERG et al., 1991). Por meio de estudo piloto foi possível chegar ao número de 18 tentativas de prática mental, correspondendo ao tempo de prática de 1 minuto, 90 tentativas para 5 minutos e 180 tentativas referentes ao tempo de 10 minutos.

\section{Procedimentos experimentais}

O pesquisador recebeu os sujeitos, que leram e assinaram um termo de consentimento livre e esclarecido. Em seguida, foram fornecidas instrução verbal e demonstração da tarefa. $\mathrm{Na}$ prática física, os sujeitos deveriam se posicionar em pé lateralmente em relação ao alvo e realizar o arremesso com a mão preferencial. A meta consistia em acertar o centro do alvo sem projetar o tronco, realizando a pegada profissional. $\mathrm{Na}$ prática mental, os sujeitos deveriam fechar os olhos e sinalizar, com a mão preferencial, o início e término de cada tentativa. Os participantes se imaginavam realizando a mesma tarefa estabelecida e explicada para a prática física. No teste de transferência novas instruções verbais e demonstrações foram informadas acerca das alterações na tarefa (mudança na distância e tipo de preensão do dardo).

\section{Resultados}

Para a análise da média do escore em blocos de 5 tentativas, uma ANOVA two-way ( 6 grupos $X$ 10 blocos) com medidas repetidas no segundo fator foi realizada para a fase de aquisição (Figura 1). Não foi detectada diferença significante no fator grupos $[F(5,66)=0,8, p=0,55]$ ou interação entre grupos e blocos $[F(45,594)=0,75, p=0,88]$. Observou-se diferença no fator blocos $[F(9$, $594)=16,6, p<0,001]$ e o teste post-hoc de Tukey mostrou que os grupos aumentaram suas pontuações do primeiro para os demais blocos de tentativas $(p<0,01)$ e do segundo, exceto $o$ primeiro bloco, para os demais blocos de tentativas $(p<0,05)$. 


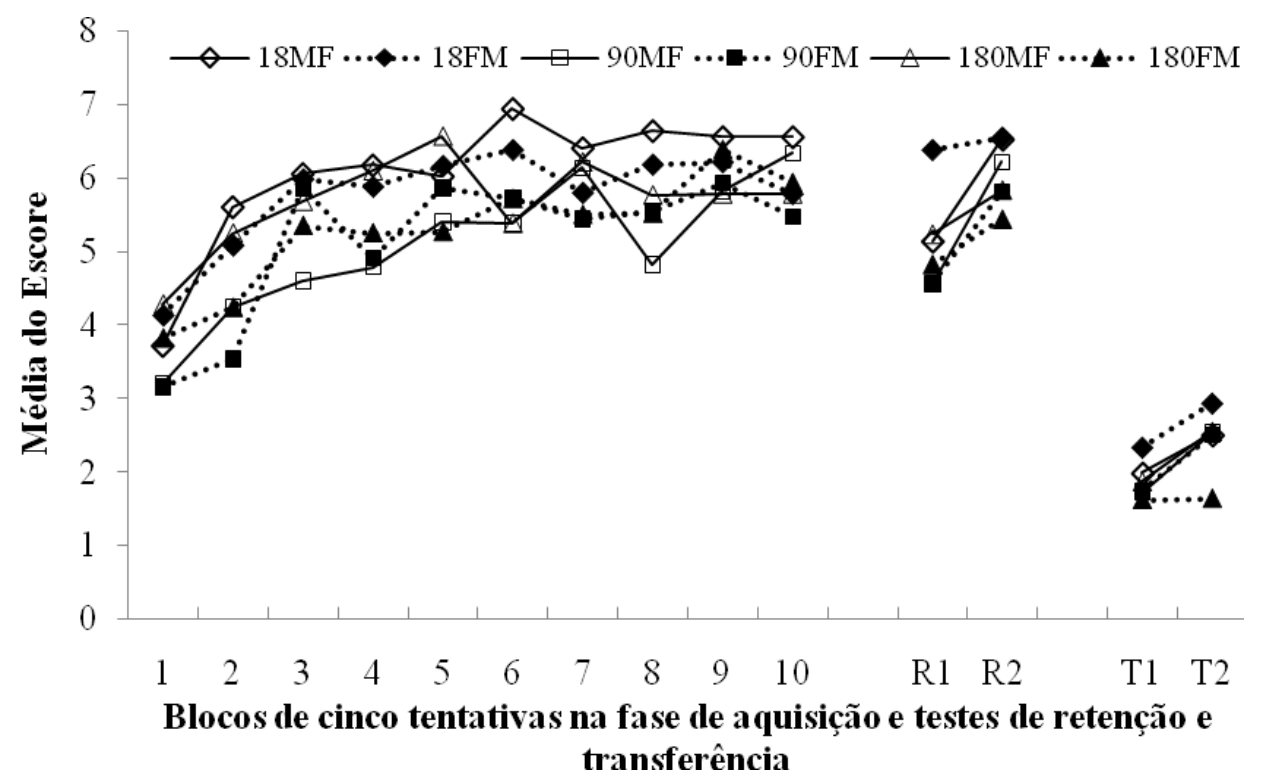

Figura 1. Média do escore na fase de aquisição (blocos 1 a 10), testes de retenção (R1 e R2) e de transferência (T1 e T2).

Outra ANOVA two-way (6 grupos $X 4$ blocos) com medidas repetidas no segundo fator foi realizada para análise dos testes de retenção e transferência, que não mostrou diferença significante no fator grupos $[F(5,66)=0,5, p=0,77]$ e na interação entre grupos e blocos $[F(15$, 198) $=0,58, \quad p=0,89]$. Observou-se diferença significante no fator blocos $[F(3,198)=158,3$, $\mathrm{p}<0,001]$ e o teste post-hoc de Tukey indicou melhor desempenho do $\mathrm{R} 2$ em relação ao $\mathrm{R} 1, \mathrm{~T} 1$ e T2, melhor desempenho de $\mathrm{R} 1$ em relação ao
T1 e T2 e melhor desempenho de T2 em relação ao $\mathrm{T} 1$.

Para o desvio padrão do escore, outra ANOVA two-way (6 grupos $X 10$ blocos) com medidas repetidas no segundo fator foi realizada para a fase de aquisição (Figura 2). Não se verificou diferença significante para grupos $[F(5,66)=0,53$, $p=0,75]$, blocos $[F(9,594)=0,86, p=0,56]$ ou interação entre grupos e blocos $[F(45,594)=1,16$, $\mathrm{p}=0,22]$.

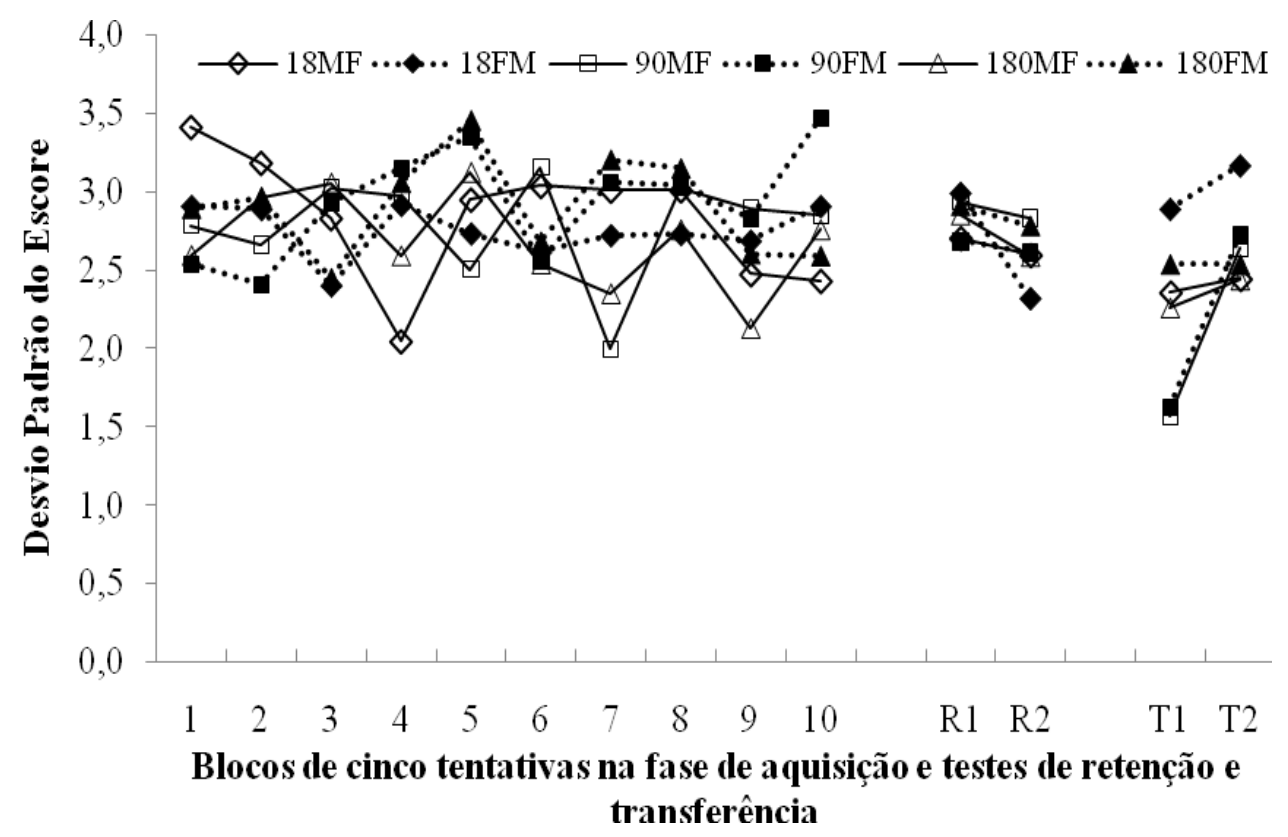

Figura 2. Desvio Padrão do escore na fase de aquisição (blocos 1 a 10), testes de retenção (R1 e R2) e de transferência (T1 e T2).

Outra ANOVA two-way (6 grupos $X 4$ blocos) com medidas repetidas no segundo fator foi realizada para análise dos blocos de tentativas dos testes. Não foi registrada diferença 
significante no fator grupos $[F(5,66)=0,46, p=0,8]$ ou interação entre grupos e blocos $[F(15$, 198) $=0,74, p=0,74]$. Observou-se diferença no fator blocos $[F(3,198)=3,31, p=0,02]$ e o teste de Tukey indicou maior variabilidade no primeiro bloco do teste de retenção (R1) em relação ao primeiro bloco do teste de transferência - T1 $(p<0,05)$.

\section{Discussão e conclusão}

O objetivo do presente estudo foi investigar os efeitos do posicionamento e quantidade de prática mental na aquisição do arremesso do dardo de salão. Os resultados mostraram similaridade entre os grupos tanto na fase de aquisição como nos testes. Assim, o seu posicionamento não foi uma variável que contribuiu para a aprendizagem do arremesso do dardo de salão. Isto confirma os achados de Weinberg et al. (1991), mas não corrobora com os resultados encontrados por Etnier e Landers (1996), em que o posicionamento da prática mental antes da prática física proporcionou desempenho superior quando comparado com o seu posicionamento anterior à prática física. Uma possível explicação para este resultado está fundamentada no nível de experiência dos participantes, que eram novatos na tarefa. A prática mental pode não ter sido efetiva quando realizada sem que os praticantes tivessem alguma idéia da habilidade, visto que uma referência mínima parece ser essencial (FELTZ; LANDERS, 1983). Por outro lado, quando se refere aos grupos em que a prática física foi realizada inicialmente, há o argumento que a prática física pode ter facilitado 0 desempenho, requerendo então menos esforço posterior (ETNIER; LANDERS, 1996). Neste caso, especula-se a possibilidade de a prática mental resultar em efeito benéfico em outras sessões de prática, o que merece ser analisado em estudos futuros.

Neste estudo, o segundo objetivo foi investigar se a quantidade de prática mental teria efeitos na aprendizagem. Os resultados mostraram que sua quantidade não interferiu na aprendizagem do arremesso de dardo de salão. Pesquisadores que investigaram a duração desta prática chegaram a diferentes resultados, com achados mostrando melhores efeitos de curtos períodos (MACKAY, 1981; RICHARDSON, 1967a) ou que o período depende das características da tarefa (WEINBERG, 1982). Por exemplo, um período mais longo é benéfico para aprendizagem de tarefas motoras (FELTZ; LANDERS, 1983), ou mesmo que não exerce influência sobre a aprendizagem motora ( $\underline{\mathrm{CAHN}}, 2008)$.

$\mathrm{Na}$ fase de aquisição foi observado que os grupos melhoraram seu desempenho a partir do segundo bloco de tentativas. Considerando que a quantidade de prática física foi a mesma para todos os grupos, pode-se especular que talvez apenas a prática física tenha sido suficiente para a melhora do desempenho dos grupos, não sendo importante a quantidade, o posicionamento, ou mesmo a própria prática mental. A ausência de diferença significante no desempenho da prática física na fase de aquisição entre os grupos que executaram prática mental antes daqueles que a realizaram após é um indicativo que não houve qualquer alteração. Esperava-se que o primeiro bloco de tentativas da prática física da fase de aquisição dos grupos que realizaram a prática mental antes apresentasse desempenho superior que os grupos que a executaram após. Diferente dos estudos de Weinberg et al. (1991) e Etnier e Landers (1996), o presente estudo utilizou sujeitos novatos como voluntários para o estudo. As pesquisas em prática mental têm utilizado sujeitos experientes e novatos na tarefa (DRISKELL et al., 1994; FELTZ; LANDERS, 1983; MAGILL, 1998; SUINN, 1993). Feltz e Landers (1983), em estudo de meta-análise, observaram a importância de um nível mínimo de habilidade dos sujeitos para a efetividade da prática mental. Contudo, os autores ressaltam que já foram encontrados resultados com sujeitos novatos na tarefa. Para Weinberg (1982) e Harris e Robinson (1986), o nível de habilidade influencia a atividade muscular durante a imaginação e esta ativação parece específica ao grupo muscular necessário à execução da tarefa, como observado por Jacobson (1932).

Entretanto, um procedimento comum utilizado por alguns pesquisadores é a submissão de sujeitos novatos a um período de familiarização da tarefa por meio de prática física (CAHN, 2008; OVERDORF et al., 2004; WOHLDMANN et al., 2008). Outro procedimento é a utilização do período de pré-teste (com prática física da habilidade) no delineamento (HIRD et al., 1991, STEBBINS, 1968; WOHLDMANN et al., 2008). Tais procedimentos podem contribuir para maior assimilação da tarefa, creditando à prática mental os efeitos de aprendizagem que ela não teria.

A semelhança entre grupos com diferentes quantidades de prática mental, ou mesmo do seu 
posicionamento na prática combinada sugere uma hipótese que para sujeitos novatos, intactos na tarefa, tal prática não contribuiu para o processo de aprendizagem motora (MACKAY, 1981; MARING, 1990; OVERDORF et al., 2004; STEBBINS, 1968; WULF et al., 1995).

É possível argumentar que a literatura indica que a prática mental é efetiva para a aprendizagem de habilidades motoras quando combinada à prática física (MACKAY, 1981; MARING, 1990; RICHARDSON, 1967a; WULF et al., 1995). O presente estudo confirmaria esta hipótese visto que todos os grupos apresentaram desempenho similar nos testes e melhoraram seu desempenho na fase de aquisição. Se os resultados não mostraram desempenho superior de uma das formas de prática combinada, também não rejeitaram os estudos que concluem em favor da similaridade entre as condições. Entretanto, ao considerar que a quantidade de prática não interferiu na aprendizagem, pode-se especular que, para sujeitos novatos sem uma familiarização à tarefa, a própria prática mental parece ser ineficiente (GOMES; UGRINOWITSCH; BENDA, 2009). Portanto, outros estudos são necessários para investigar questões ainda inconclusivas, e principalmente testar a hipótese se a prática física, mesmo como familiarização à tarefa, é essencial para uma aprendizagem motora mais efetiva.

\section{Referências}

CAHN, D. The effects of varying ratios of physical and mental practice, and task difficulty on performance of a tonal pattern. Psychology of Music, Thousand Oaks, v. 36, p. 179-191, 2008.

DENIS, M. Visual imagery and the use of mental practice in the development of motor skills.

Canadian Journal of Applied Sports Sciences, Vanier, v. 10, p. 4-6, 1985.

DRISKELL, J. E.; COPPER, C.; MORAN, A. Does mental practice enhance performance? Journal of Applied Psychology, Stanford, v. 79, p. 481492, 1994.

ETNIER, J. L.; LANDERS, D. M. The influence of procedural variables on the efficacy of mental practice. The Sport Psychologist, Champaign, v. 10, p. 48-57, 1996.

FELTZ, D. L.; LANDERS, D. M. The effects of mental practice on motor skill learning and performance: a meta-analysis. Journal of Sport Psychology, Champaign, v. 5, p. 25-57, 1983.
GOMES, T. V. B.; UGRINOWITSCH, H.; BENDA, R. N. Effects of mental practice in novice motor skill acquisition. Journal of Sport and Exercise Psychology, Champaign, v. 31, p. S65, 2009.

HARRIS, D. V.; ROBINSON, W. J. The effects of skill level on EMG activity during internal and external imagery. Journal of Sport Psychology, Champaign, v. 8, p. 105-111, 1986.

HIRD, J. S.; LANDERS, D. M.; THOMAS, J. R.; HORAN, J. J. Physical practice is superior to mental practice in enhancing cognitive and motor task performance. Journal of Sport \& Exercise Psychology, Champaign, v. 8, p. 281-293, 1991.

JACOBSON, R. Electrophysiology of mental activities. American Journal of Psychology, Illinois, v. 44, p. 677-694, 1932.

LANDERS, D. M.; LANDERS, D. M. Teacher versus peer models: effects of model's presence and performance level on motor behavior.

Journal of Motor Behavior, Washington, v. 5, p. 129-139, 1973.

MACKAY, D. G. The problem of rehearsal or mental practice. Journal of Motor Behavior, Washington, v. 13, p. 274-285, 1981.

MAGILL, R. A. Motor Learning: concepts and applications. Boston: WCB/McGraw-Hill, 1998.

MARING, J. R. Effects of mental practice on rate of skill acquisition. Physical Therapy, New York, v. 70, p. $165-172,1990$.

MILLARD, M.; MAHONEY, C.; WARDROP, J. A preliminary study of mental and physical practice on the kayak wet exit skill. Perceptual and Motor Skills, Missoula, v. 92, p. 977-984, 2001.

OVERDORF, V.; SCHWEIGHARDT, R.; PAGE, S. J.; McGRATH, R. E. Mental and physical practice schedules in acquisition and retention of novel timing skills. Perceptual and Motor Skills, Missoula, v. 99, p. 51-62, 2004.

RICHARDSON, A. Mental practice: a review and discussion (Part 1). Research Quartely, Washington, v. 38, p. 95-107, 1967a.

RICHARDSON, A. Mental practice: a review and discussion (Part 2). Research Quartely, Washington, v. 38, p. 263-273, 1967b.

STEBBINS, J. R. A comparison of the effects of physical and mental practice in learning a motor skill. Research Quarterly, Washington, v. 39, p. 714-220, 1968.

SUINN, R. Imagery. In: SINGER, R. N.; MURPHEY, M.; TENNANT, L. K. Handbook of 
research on sport psychology. New York:

Macmillan Publisher Company, p. 492-510, 1993.

WEINBERG, R. S. The relationship between mental preparation strategies and motor performance: a review and critique. Quest, Champaign, v. 33, p. 195-213, 1982.

WEINBERG, R.; HANKES, D.; JACKSON, A. Effect of the length and temporal location of the mental preparation interval on basketball shooting performance. International Journal of Sport Psychology, Florida, v. 22, p. 3-14, 1991.

WOHLDMANN, E. L.; HEALY, A. F.; JUNIOR, L. E. B. A mental practice superiority effect: less retroactive interference and more transfer than physical practice. Journal of Experimental Psychology: learning memory and cognition, Washington, v. 34, p. 823-833, 2008.

WULF, G.; HORSTMANN, G.; CHOI, B. Does mental practice work like physical practice without information feedback? Research Quarterly for Exercise and Sport, Washington, v. 66, p. 262267, 1995.

\section{Endereço:}

Rodolfo Novellino Benda

Escola de Educação Física, Fisioterapia e Terapia Ocupacional - UFMG

Av. Pres. Antônio Carlos, 6627 - Pampulha

Belo Horizonte MG Brasil

31270-901

Telefone: (31) 3409-2394 3409-2325

e-mail: rodolfobenda@yahoo.com.br

Recebido em: 15 de setembro de 2011. Aceito em: 20 de dezembro de 2011.

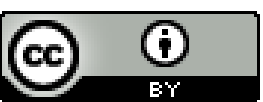

Motriz. Revista de Educação Física. UNESP, Rio Claro, SP, Brasil - elSSN: 1980-6574 - está licenciada sob Creative Commons - Atribuição 3.0 